\title{
General local and rectilinear vibrational coordinates consistent with Eckart's conditions
}

\author{
Iván Scivetti, ${ }^{1}$ Jorge Kohanoff, ${ }^{1}$ and Nikitas I. Gidopoulos ${ }^{2}$ \\ ${ }^{1}$ Atomistic Simulation Centre, Queen's University Belfast, Belfast, BT7 1NN Northern Ireland, United Kingdom \\ ${ }^{2}$ ISIS Facility, STFC, Rutherford Appleton Laboratory, Harwell Science and Innovation Campus, Didcot, Oxon OX11 OQX, \\ England, United Kingdom
}

(Received 21 January 2009; published 24 March 2009)

\begin{abstract}
We present a general method to construct a set of local rectilinear vibrational coordinates for a nonlinear molecule whose reference structure does not necessarily correspond to a stationary point of the potentialenergy surface. We show both analytically and with a numerical example that the vibrational coordinates satisfy Eckart's conditions. In addition, we find that the Watson Hamiltonian provides a fairly robust description even of highly excited vibrational states of triatomic molecules, except for a few states of large amplitude motion sampling the singular region of the Hamiltonian. These states can be identified through slow convergence.
\end{abstract}

DOI: 10.1103/PhysRevA.79.032516

PACS number(s): 33.15.-e, 31.15.-p, 33.20.Wr

\section{INTRODUCTION}

In the rovibrational description of molecular systems, the use of local rectilinear vibrational coordinates constitutes one possible choice for describing the nuclear degrees of freedom. In contrast to curvilinear internal coordinates (usually, bond lengths and angles), the definition of local rectilinear coordinates requires the choice of a reference molecular structure. Within this framework, the Watson (or WatsonEckart) Hamiltonian [1,2] provides a general description of the rovibrational problem of any molecule.

Normal coordinates, calculated at a given equilibrium structure, arise as a possible choice of local rectilinear vibrational coordinates. However, this is not the only choice. In fact, for any reference molecular structure (not necessarily an equilibrium one), Eckart's conditions $[3,4]$ define a frame of axes that rotates together with the molecule. In this rotating frame, departures from the reference structure are purely due to vibrations. In addition, the definition of this local rectilinear vibrational space depends on the reference molecular structure (from now on, reference structure).

If the Watson Hamiltonian is solved exactly, the initial choice of the reference structure is immaterial as the full solution of the rovibrational problem from any reference structure must give the same result. Therefore, there is an appealing independence on the choice of the reference structure. In practice, the latter is invariably chosen to be the classical equilibrium structure or at least a stationary point of the potential-energy surface (PES) [5].

Nevertheless, there is an optimal reference structure from which numerically solving the problem becomes most efficient. This structure should be the quantum-averaged one since vibrational departures from this reference structureand consequently the Coriolis coupling, i.e., the interaction between vibrational and rotational motions-is minimal [6]. This is the case of large-amplitude-of-motion molecules such as ammonia, where the saddle point arises as the most convenient reference structure to describe the rovibrational problem [7]. In addition, approximate forms of the Watson Hamiltonian are quite often used to describe vibrational spectra of larger molecules with zero total angular momen- tum $(J=0)[8-10]$. Another example of approximation might involve the expansion of the PES to second order around an appropriately chosen reference structure. Away from equilibrium, one would have to use Eckart's conditions to separate out rotations, otherwise, such approximate harmonic PES's would not be invariant under rigid rotations. In all these cases, the choice of the reference structure transcends efficiency matters. In effect, an inappropriate choice of the reference structure may potentially lead to results that are quantitatively incorrect or perhaps even qualitatively in cases of extreme anharmonicity [11]. To investigate these issues it is necessary to employ Eckart's conditions [3] at an arbitrary reference structure.

We are not aware of other calculations in the literature using the Watson Hamiltonian at reference structures away from stationary points of the PES. In this work, we present a general method to construct local rectilinear vibrational coordinates for any nonlinear molecular reference structure. We demonstrate analytically that the vibrational coordinates we construct satisfy Eckart's conditions. We then study the case of water molecule (a molecule with rather weak anharmonicity) and demonstrate numerically that the full solution of the rovibrational problem for $J=0$ from three different reference structures is the same.

In a forthcoming publication [11], we shall focus on the effect of choosing different reference structures when the problem is approximately described by neglecting the rovibrational terms of the Watson Hamiltonian.

\section{FORMALISM}

\section{A. Center-of-mass and relative coordinates}

We start by assuming the Born-Oppenheimer approximation to separate the electronic and nuclear coordinates [12]. The electronic energy as a function of the nuclear positions $\left\{\mathbf{R}_{1}, \ldots, \mathbf{R}_{N}\right\}$ provides the PES in which the nuclei move. In the absence of an external field, translating the positions of all nuclei by the same vector a does not modify the electronic energy. Similarly, rotating all the nuclei rigidly about their center-of-mass (c.m.) does not alter the electronic en- 
ergy either. We shall first separate the nuclear c.m., reducing the number of nuclear degrees of freedom from $3 N$ to $3 N$ -3 . Then, we shall transform to rectilinear infinitesimal vibrations ( $3 N-6$ degrees) and rigid rotations (3 degrees).

Let us consider a molecule made of $N$ nuclei of masses $M_{1}, \ldots, M_{N}$ and charges $Z_{1}, \ldots, Z_{N}$ at positions $\left\{\mathbf{R}_{1}, \ldots, \mathbf{R}_{N}\right\}$ which are Cartesian coordinates given in an inertial laboratory frame of reference. In what follows, the dummy index $n$ takes values between 1 and $N$, while the dummy indices $\mu, \nu$ vary between 1 and $N-1$. We now transform the Cartesian coordinates to relative and c.m. coordinates,

$$
\left\{\mathbf{R}_{1}, \ldots, \mathbf{R}_{N}\right\} \rightarrow\left\{\mathbf{Q}_{1}, \ldots, \mathbf{Q}_{N-1} ; \mathbf{R}_{\mathrm{c} . \mathrm{m} .}\right\},
$$

with

$$
\begin{gathered}
\mathbf{R}_{\text {c.m. }}=\frac{1}{M_{\text {c.m. } n=1}} \sum_{n}^{N} M_{n} \mathbf{R}_{n}, \\
\mathbf{Q}_{\mu}=\sum_{n=1}^{N} \lambda_{\mu n} \mathbf{R}_{n}, \quad \mu=1, \ldots, N-1,
\end{gathered}
$$

where $M_{\text {c.m. }}=\sum_{n=1}^{N} M_{n}$. The relative nuclear coordinates $\mathbf{Q}_{\mu}$ are not unique, but any choice should remain invariant under rigid translations of the molecular system, i.e., for every coordinate $\mu$ and any translation $\mathbf{a}$,

$$
\sum_{n=1}^{N} \lambda_{\mu n}\left(\mathbf{R}_{n}+\mathbf{a}\right)=\mathbf{Q}_{\mu}
$$

This imposes a constraint on the coefficients $\lambda_{\mu n}$ that is

$$
\sum_{n=1}^{N} \lambda_{\mu n}=0
$$

This last equation is completely equivalent to the first condition introduced by Eckart [3] to account for translational invariance. The coefficients $\lambda_{\mu n}$ are chosen in such a way that the Jacobian of the transformation described by expressions (1)-(3) is unity. The relative coordinates $\left\{\mathbf{Q}_{1}, \ldots, \mathbf{Q}_{N-1}\right\}$ are independent and form a basis in the space of coordinates that are invariant under translations. In particular, any other linear combination of $\left\{\mathbf{R}_{1}, \ldots, \mathbf{R}_{N}\right\}$ having the same property can be expressed as a linear combination of the elements of this basis. In particular

$$
\mathbf{R}_{n}-\mathbf{R}_{\mathrm{c} . \mathrm{m} .}=\sum_{\mu=1}^{N-1} \gamma_{\mu n} \mathbf{Q}_{\mu}
$$

Equation (6) is the inverse of the transformation [Eqs. (1)-(3)]. Multiplying Eq. (6) by $M_{n}$ and summing over $n$ we find

$$
\sum_{\mu=1}^{N-1} \mathbf{Q}_{\mu} \sum_{n=1}^{N} M_{n} \gamma_{\mu n}=0
$$

and since $\mathbf{Q}_{\mu}$ are independent, we obtain

$$
\sum_{n=1}^{N} M_{n} \gamma_{\mu n}=0
$$

which is an alternative expression to Eq. (5) for the first set of Eckart's conditions.

The PES, $\mathcal{E}$, is independent of $\mathbf{R}_{\text {c.m. }}$ and can be expressed in terms of the relative coordinates

$$
\mathcal{E}=\mathcal{E}\left(\mathbf{Q}_{1}, \ldots, \mathbf{Q}_{N-1}\right) .
$$

The molecular Hamiltonian in relative coordinates, i.e., after separating the c.m., takes the following form:

$$
\mathcal{H}=-\frac{\hbar^{2}}{2} \sum_{\mu, \kappa=1}^{N-1} \beta_{\mu \kappa} \nabla_{\mathbf{Q}_{\mu}} \cdot \nabla_{\mathbf{Q}_{\kappa}}+\mathcal{E}\left(\mathbf{Q}_{1}, \ldots, \mathbf{Q}_{N-1}\right),
$$

where

$$
\beta_{\mu \kappa}=\sum_{n=1}^{N} \frac{\lambda_{\mu n} \lambda_{\kappa n}}{M_{n}}
$$

The choice of relative coordinates given by Eq. (3) is convenient when the matrix $\beta_{\mu \kappa}$ is diagonal. Therefore, without loss of generality we shall restrict our study to transformations to relative coordinates that lead to diagonal $\beta_{\mu \kappa}$. In such a case the inverse of $\beta_{\kappa \kappa} \geq 0$ becomes the effective mass $m_{\kappa}$ of $\mathbf{Q}_{\kappa}$,

$$
\beta_{\mu \kappa}=\frac{\delta_{\mu \kappa}}{m_{\kappa}} .
$$

In Appendix A we show that condition (12) leads to

$$
\sum_{n=1}^{N} M_{n} \gamma_{\mu n} \gamma_{\kappa n}=m_{\kappa} \delta_{\mu \kappa},
$$

which will be used later on to simplify the expression for the second set of Eckart's conditions. In Appendix B we describe a general way to obtain a transformation to relative coordinates that leads to diagonal $\beta_{\mu \kappa}$.

\section{B. Rotating frame of reference}

The relative coordinates $\left\{\mathbf{Q}_{1}, \ldots, \mathbf{Q}_{N-1}\right\}$ are linear combinations of the Cartesian coordinates $\left\{\mathbf{R}_{1}, \ldots, \mathbf{R}_{N}\right\}$, both given in the laboratory frame of reference. With the separation of the three translational degrees of freedom of the molecule, the remaining $(3 N-3)$ degrees correspond to rotations and vibrations. Now we separate the three rotations from the $(3 N-6)$ vibrations. Here, we opt to use local rectilinear vibrational and rotational coordinates, similar to the definition of normal modes. At variance with internal coordinates such as distances and angles, or the more general Jacobi vectors, in these coordinates the separation between rotations and vibrations is not global but depends on the reference structure of the molecule. Within this framework, it is useful to consider a rotating frame of reference attached to the molecule (also called body-fixed frame).

We denote by $\mathbf{x}, \mathbf{y}, \mathbf{z}$ the unit vectors of the laboratory frame of reference and by $\widetilde{\mathbf{x}}, \widetilde{\mathbf{y}}, \widetilde{\mathbf{z}}$ the unit vectors in the rotating frame of reference. For now, the axes of the rotating 
frame are free, but later on they will be chosen to coincide with the principal axes of inertia in the reference structure. The relative coordinates can be expressed in the rotating frame or in the laboratory frame. Let $\mathcal{R}$ represent a rotation about the c.m. We have

$$
\begin{aligned}
\left(\begin{array}{c}
\tilde{\mathbf{x}} \\
\tilde{\mathbf{y}} \\
\tilde{\mathbf{z}}
\end{array}\right) & =\mathcal{R}^{-1}\left(\begin{array}{l}
\mathbf{x} \\
\mathbf{y} \\
\mathbf{z}
\end{array}\right), \\
\left(\begin{array}{l}
\mathbf{x} \\
\mathbf{y} \\
\mathbf{z}
\end{array}\right) & =\mathcal{R}\left(\begin{array}{c}
\tilde{\mathbf{x}} \\
\tilde{\mathbf{y}} \\
\tilde{\mathbf{z}}
\end{array}\right) .
\end{aligned}
$$

With this, $Q_{\mu, x}, Q_{\mu, y}$, and $Q_{\mu, z}$ are the projections of $\mathbf{Q}_{\mu}$ onto the axes of the laboratory frame, and $Q_{\mu, \tilde{x}}, Q_{\mu, \tilde{y}}$, and $Q_{\mu, \tilde{z}}$ are the projections of $\mathbf{Q}_{\mu}$ onto the rotating axes of the rotating frame. In the literature it is customary to choose the rotational coordinates to be the three Euler angles that determine the rotating frame. The rectilinear vibrational coordinates that we are going to construct satisfy the Eckart's conditions and can be used together with the three Euler angles to describe the full rovibrational problem using the Watson Hamiltonian.

\section{Eckart's conditions}

The Eckart's conditions have been proposed as a means to define the rotating frame. However, this is rarely done in practice (see below). The rotating frame of reference is defined for some molecular geometry, which is specified by the reference values of the relative coordinates $\mathbf{Q}_{\mu}^{0}$. This reference geometry (reference structure), which in principle is arbitrary, rotates in the laboratory frame but does not rotate in the rotating frame. The definition of a set of rectilinear vibrational coordinates depends on the reference geometry which, almost invariably, is chosen as the classical equilibrium structure at the minimum of the PES. This choice is clearly consistent but by no means necessary. The aim of this work is to use Eckart's conditions in order to obtain local, rectilinear vibrational coordinates at arbitrary reference structures. In order to test our approach and to demonstrate how it can be used in practice, we shall solve the exact Watson Hamiltonian for some arbitrary reference geometries to confirm that the different solutions coincide.

The second set of Eckart's conditions imposes that, for a given reference configuration $\mathbf{Q}_{\mu}^{0}$, departures of the form $\mathbf{Q}_{\mu}-\mathbf{Q}_{\mu}^{0}$ represent vibrations if

$$
\sum_{n=1}^{N} M_{n}\left(\sum_{\mu=1}^{N-1} \gamma_{\mu n} \mathbf{Q}_{\mu}^{0}\right) \times\left(\sum_{\kappa=1}^{N-1} \gamma_{\kappa n} \mathbf{Q}_{\kappa}\right)=0
$$

Using Eq. (13), the second Eckart condition simplifies to

$$
\sum_{\mu=1}^{N-1} m_{\mu} \mathbf{Q}_{\mu}^{0} \times \mathbf{Q}_{\mu}=0 .
$$

It is customary to express the Eckart's conditions explicitly in the rotating frame. However, since the cross product of two vectors is rotationally invariant, i.e., for any two vectors $\mathbf{u}, \mathbf{v}$ we have $(\mathcal{R} \mathbf{u}) \times(\mathcal{R} \mathbf{v})=\mathcal{R}(\mathbf{u} \times \mathbf{v})$, the Eckart's conditions hold independently of which set of coordinate axes are used to project the vectors $\mathbf{Q}_{\mu}$.

In the literature, the physical meaning of the Eckart's conditions is somewhat unclear. For example, in page 275 of Wilson et al. [13] we read "... this is almost but not quite equivalent to stating that there must be no angular momentum with respect to the rotating system of axes." Here we shall show that Eckart's conditions imply that the rotating frame of reference is determined by defining rotations as those transformations which do not alter bond lengths and internal angles of the molecule.

Further to the transformation to relative coordinates, we make a transformation to mass-scaled coordinates $\left\{\mathbf{q}_{1}, \ldots, \mathbf{q}_{N-1}\right\}$, all of them bearing the same mass $M$,

$$
\mathbf{q}_{\kappa}=\sqrt{\frac{m_{\kappa}}{M}} \mathbf{Q}_{\kappa} .
$$

By requiring that the Jacobian of the mass-scaling transformation is unity, we find for the common mass

$$
M^{N-1}=\prod_{\kappa=1}^{N-1} m_{\kappa} \text {. }
$$

In these set of coordinates, the Eckart's condition (17) reads as

$$
M \sum_{\mu=1}^{N-1} \mathbf{q}_{\mu}^{0} \times \mathbf{q}_{\mu}=0 .
$$

In conclusion, given a reference structure $\mathbf{q}_{\mu}^{0}$, the space of local rectilinear vibrations is defined by all the departures $\mathbf{q}_{\mu}-\mathbf{q}_{\mu}^{0}$ that satisfy Eq. (20).

\section{Rectilinear infinitesimal rotations and vibrations}

The $3 N-3$ coordinates $\left\{q_{\mu \alpha}\right\}$ in the laboratory frame of reference $(\alpha=x, y, z)$ and in rotating frame of reference $(\alpha$ $=\tilde{x}, \tilde{y}, \widetilde{z})$ have mixed vibrational and rotational characters. We shall construct local rotational and vibrational coordinates that are linear forms of the coordinates $q_{\mu \alpha}, \mu$ $=1, \ldots, N-1$. To this end, it is convenient to consider the vector space $\mathcal{V}$ of all such linear forms. This is a $3 N-3$-dimensional space that includes vibrations $(3 N-6$ dimensions) and rotations (three dimensions).

Our aim in this work is to find an orthogonal transformation to a new set of coordinates $\left\{\zeta_{x}^{\text {rot }}, \zeta_{y}^{\text {rot }}, \zeta_{z}^{\text {rot }}\right\}$ and $\left\{\zeta_{1}, \zeta_{2}, \ldots, \zeta_{3 N-6}\right\}$ that correspond to rectilinear infinitesimal pure rotations and vibrations, i.e., vibrational coordinates that do not show any rotational character, and vice versa. The use of rectilinear coordinates implies that the division between rotations and vibrations in $\mathcal{V}$ is not global but local, i.e., for a given molecule it depends on the reference structure of the molecule. For example, a coordinate representing an infinitesimal rotation for a molecule when the reference structure is taken at the minimum energy configuration will most likely acquire some vibrational character when a different reference structure is considered, e.g., the saddle point 
configuration, or another local minimum energy structure.

\section{Construction of coordinate invariant under rotations}

In the rotating frame of reference we choose a reference configuration specified in terms of the nuclear mass-scaled relative coordinates $\mathbf{q}_{\kappa}^{0}$. We consider the space $\mathcal{V}^{\text {vibr }}$ of linear forms of $q_{\kappa \alpha}$ that remain invariant under small, arbitrary rigid rotations of the molecule. The space of rotations $\mathcal{V}^{\text {rot }}$ is orthogonal to $\mathcal{V}^{\text {vibr }}$. In Sec. II D 3 we shall consider the space of rotations $\mathcal{V}^{\text {rot }}$ as defined by Eckart and verify that, indeed, every vector in $\mathcal{V}^{\text {rot }}$ is orthogonal to every vector in $\mathcal{V}^{\text {vibr }}$. This will prove that the present construction of vibrational coordinates is consistent with the Eckart conditions.

To proceed, we introduce small arbitrary changes in the coordinates

$$
\mathbf{q}_{\kappa}=\mathbf{q}_{\kappa}^{0}+\mathbf{u}_{\kappa},
$$

where $\left\|\mathbf{u}_{\kappa}\right\| \ll 1$. We now take the case where $\mathbf{u}_{\kappa}$ represent small rotations of the molecule as a whole. Since $\mathbf{q}_{\kappa}$ are relative vectors, their magnitudes and relative angles do not change by a rotation. Therefore, rotations can be defined as transformations which do not change bond lengths and internal angles. Mathematically, we express this by requiring that rigid rotations leave invariant the inner products

$$
\mathbf{q}_{\kappa} \cdot \mathbf{q}_{\lambda}=\mathbf{q}_{\kappa}^{0} \cdot \mathbf{q}_{\lambda}^{0}
$$

and to first order in $\left\|\mathbf{u}_{\kappa}\right\|$,

$$
\mathbf{q}_{\kappa}^{0} \cdot \mathbf{u}_{\lambda}+\mathbf{u}_{\kappa} \cdot \mathbf{q}_{\lambda}^{0}=0
$$

or, equivalently,

$$
\mathbf{q}_{\kappa}^{0} \cdot \mathbf{q}_{\lambda}+\mathbf{q}_{\kappa} \cdot \mathbf{q}_{\lambda}^{0}-2 \mathbf{q}_{\kappa}^{0} \cdot \mathbf{q}_{\lambda}^{0}=0 .
$$

Therefore, to first order, the linear forms of the dynamical coordinates $\left\{\mathbf{q}_{\kappa}\right\}$,

$$
\begin{gathered}
\zeta_{\kappa}^{\mathrm{vibr}}=\mathbf{q}_{\kappa}^{0} \cdot \mathbf{q}_{\kappa}, \\
\zeta_{\kappa \lambda}^{\mathrm{vibr}}=\mathbf{q}_{\kappa}^{0} \cdot \mathbf{q}_{\lambda}+\mathbf{q}_{\lambda}^{0} \cdot \mathbf{q}_{\kappa}, \quad \kappa \neq \lambda,
\end{gathered}
$$

remain unchanged under infinitesimal rigid rotations, and consequently, they represent vibrational coordinates. The linear forms [Eq. (22)] span the $(3 N-6)$-dimensional space of vibrations $\mathcal{V}^{\text {vibr }}$. Moreover, these vibrational coordinates are the same in both the rotating and the laboratory frames since the inner product is invariant under rotations. The number of linear forms in [Eq. (22)] is $N(N-1) / 2$ but not all of them are independent when $N>4$; the number of internal coordinates of a nonlinear molecule are $3 N-6$ and $N(N-1) / 2$ $\geq 3 N-6$ (the equality sign holds for $N=3,4$ ).

When the reference structure is linear, the relative reference coordinates are all multiples of a common vector parallel to the axis of the molecule, $\mathbf{q}_{\kappa}^{0}=c_{\kappa} \mathbf{q}^{0}$, where $c_{\kappa}$ is a constant. In this case the vibrational coordinates defined above become $\zeta_{\kappa}^{\text {vibr }}=c_{\kappa} \mathbf{q}^{0} \cdot \mathbf{q}_{\kappa}$ and $\zeta_{\kappa \lambda}^{\text {vibr }}=c_{\kappa} \mathbf{q}^{0} \cdot \mathbf{q}_{\lambda}+c_{\lambda} \mathbf{q}^{0} \cdot \mathbf{q}_{\kappa}$. Therefore, for a linear molecule, the coordinates $\zeta_{\kappa \lambda}^{\text {vibr }}$ depend linearly on $\zeta_{\lambda}^{\text {vibr }}$ and $\zeta_{\kappa}^{\text {vibr }}$, and the set of vibrational coordinates [Eq. (22)] does not span the whole $(3 N-5)$-dimensional vibrational space but only a subspace of dimensionality $N-1$. In the following we exclude linear molecules from our study.

\section{Rotational coordinates from Eckart's conditions}

The second Eckart condition [Eq. (20)] specifies three degrees of freedom that span the space of infinitesimal rotations $\mathcal{V}^{\text {rot }} \subset \mathcal{V}$,

$$
\boldsymbol{\zeta}^{\text {rot }}=\sqrt{M} \sum_{\mu=1}^{N-1} \mathbf{q}_{\mu}^{0} \times \mathbf{q}_{\mu},
$$

where the square root of the mass is introduced to aid normalization of the rotational coordinates. In fact, since any vibrational coordinates must satisfy $\sum_{\mu=1}^{N-1} \mathbf{q}_{\mu}^{0} \times \mathbf{q}_{\mu}=0$, any coordinate that satisfies $\sum_{\mu=1}^{N-1} \mathbf{q}_{\mu}^{0} \times \mathbf{q}_{\mu} \neq 0$ must be a rotational coordinate. Therefore, such three independent degrees of freedom are fixed by condition (23);

$$
\begin{aligned}
& \zeta_{x}^{\text {rot }}=\sqrt{M} \sum_{\mu=1}^{N-1}\left(q_{\mu y}^{0} q_{\mu z}-q_{\mu z}^{0} q_{\mu y}\right), \\
& \zeta_{y}^{\text {rot }}=\sqrt{M} \sum_{\mu=1}^{N-1}\left(q_{\mu z}^{0} q_{\mu x}-q_{\mu x}^{0} q_{\mu z}\right), \\
& \zeta_{z}^{\text {rot }}=\sqrt{M} \sum_{\mu=1}^{N-1}\left(q_{\mu x}^{0} q_{\mu y}-q_{\mu y}^{0} q_{\mu x}\right) .
\end{aligned}
$$

It is useful at this point to consider an orthonormal basis in the $(3 N-3)$-dimensional vector space $\mathcal{V}$ given by $q_{\mu \alpha}$. We associate each element $q_{\kappa a}$ with a basis vector $\left|q_{\kappa \alpha}\right\rangle$ of $\mathcal{V}$ represented by

$$
\begin{aligned}
\left|q_{1 x}\right\rangle & =\left(\begin{array}{c}
1 \\
0 \\
\vdots \\
0 \\
0
\end{array}\right), \quad\left|q_{1 y}\right\rangle=\left(\begin{array}{c}
0 \\
1 \\
\vdots \\
0 \\
0
\end{array}\right), \\
\left|q_{(N-1) y}\right\rangle & =\left(\begin{array}{c}
0 \\
0 \\
0 \\
\vdots \\
1 \\
0
\end{array}\right), \quad\left|q_{(N-1) z}\right\rangle=\left(\begin{array}{c}
0 \\
0 \\
\vdots \\
0 \\
1
\end{array}\right) .
\end{aligned}
$$

Note that the reference positions $q_{\kappa \alpha}^{0}$ are scalars and not vectors of $\mathcal{V}$.

Using the notation of the vector space, the infinitesimal rectilinear vibrations can be written as

$$
\begin{aligned}
& \left\langle\zeta_{\kappa}^{\mathrm{vibr}}\right|=(\underbrace{0,0,0}_{1}, \ldots, \underbrace{q_{\kappa x}^{0}, q_{\kappa y}^{0}, q_{\kappa z}^{0}}_{\kappa}, \ldots, \underbrace{0,0,0}_{N-1}), \\
& \left\langle\zeta_{\kappa \lambda}^{\mathrm{vibr}}\right|=(\underbrace{0,0,0, \ldots,}_{1}, \underbrace{q_{\lambda x}^{0}, q_{\lambda y}^{0}, q_{\lambda z}^{0}}_{\kappa}, \ldots, \underbrace{q_{\kappa x}^{0}, q_{\kappa y}^{0}, q_{\kappa z}^{0}}_{\lambda}, \ldots \underbrace{0,0,0)}_{N-1},
\end{aligned}
$$


while the infinitesimal rectilinear rotations read as

$$
\begin{aligned}
& \left.\frac{\left|\zeta_{x}^{\mathrm{rot}}\right\rangle}{\sqrt{M}}=\left(\begin{array}{c}
0 \\
-q_{1 z}^{0} \\
q_{1, y}^{0} \\
0 \\
-q_{2 z}^{0} \\
q_{2, y}^{0} \\
\vdots \\
0 \\
-q_{(N-1) z}^{0} \\
q_{(N-1) y}^{0}
\end{array}\right), \quad \frac{\left|\zeta_{y}^{\mathrm{rot}}\right\rangle}{\sqrt{M}}=\mid \begin{array}{c}
q_{1 z}^{0} \\
0 \\
-q_{1, x}^{0} \\
q_{2 z}^{0} \\
0 \\
-q_{2, x}^{0} \\
\vdots \\
q_{(N-1) z}^{0} \\
0 \\
-q_{(N-1) x}^{0}
\end{array}\right), \\
& \frac{\left|\zeta_{z}^{\text {rot }}\right\rangle}{\sqrt{M}}=\left|\begin{array}{c}
-q_{1 y}^{0} \\
q_{1, x}^{0} \\
0 \\
-q_{2 y}^{0} \\
q_{2, x}^{0} \\
0 \\
\vdots \\
-q_{(N-1) y}^{0} \\
q_{(N-1) x}^{0} \\
0
\end{array}\right|
\end{aligned}
$$

The inner products between the rectilinear infinitesimal rotations are given by $(\alpha, \beta)=x, y, z$

$$
\begin{aligned}
\left\langle\zeta_{\alpha}^{\mathrm{rot}} \mid \zeta_{\alpha}^{\mathrm{rot}}\right\rangle & =M \sum_{\mu=1}^{N-1}\left[\left(q_{\mu}^{0}\right)^{2}-\left(q_{\mu \alpha}^{0}\right)^{2}\right] \\
& =\sum_{\mu, \kappa=1}^{N-1} \delta_{\mu \kappa} m_{\mu}\left[\mathbf{Q}_{\mu}^{0} \cdot \mathbf{Q}_{\kappa}^{0}-Q_{\mu \alpha}^{0} Q_{\kappa \alpha}^{0}\right] \\
& =\sum_{n=1}^{N} M_{n} \sum_{\mu, \kappa=1}^{N-1} \gamma_{\mu n} \gamma_{\kappa n}\left[\mathbf{Q}_{\mu}^{0} \cdot \mathbf{Q}_{\kappa}^{0}-Q_{\mu \alpha}^{0} Q_{\kappa \alpha}^{0}\right] \\
& =\sum_{n=1}^{N} M_{n}\left\{\mid\left(\mathbf{R}_{n}-\left.\mathbf{R}_{\mathrm{c} . \mathrm{m} .)_{0}}\right|^{2}-\left[\left(R_{n \alpha}-R_{\mathrm{c} . \mathrm{m} . \alpha}\right)_{0}\right]^{2}\right\}=I_{\alpha \alpha}^{0}\right.
\end{aligned}
$$

and for $\alpha \neq \beta$,

$$
\begin{aligned}
\left\langle\zeta_{\alpha}^{\mathrm{rot}} \mid \zeta_{\beta}^{\mathrm{rot}}\right\rangle & =-M \sum_{\mu=1}^{N-1} q_{\mu \alpha}^{0} q_{\mu \beta}^{0}=-\sum_{\mu, \kappa=1}^{N-1} \delta_{\mu \kappa} m_{\mu} Q_{\mu \alpha}^{0} Q_{\kappa \beta}^{0} \\
& =-\sum_{n=1}^{N} M_{n} \sum_{\mu, \kappa=1}^{N-1} \gamma_{\mu n} \gamma_{\kappa n} Q_{\mu \alpha}^{0} Q_{\kappa \beta}^{0} \\
& =-\sum_{n=1}^{N} M_{n}\left(R_{n \alpha}-R_{\text {c.m. } \alpha}\right)_{0}\left(R_{n \beta}-R_{\text {c.m. } \beta}\right)_{0}=I_{\alpha \beta}^{0} .
\end{aligned}
$$

The quantities $I_{\alpha \beta}^{0}$ are the components of the inertia tensor for the reference structure relative to the c.m. Until now, we have not made a choice for the orientation of the reference configuration $\left\{\mathbf{q}_{1}^{0}, \ldots, \mathbf{q}_{N-1}^{0}\right\}$ in the rotating frame. In fact, there is a three-dimensional continuum of equivalent configurations. Here, we choose the particular orientation such that the principal axes of inertia lie along the coordinate axes of the rotating frame of reference. With this choice the inertia tensor is diagonal and, according to Eq. (30), the rectilinear infinitesimal rotations $\zeta_{\alpha}^{\text {rot }}$ are mutually orthogonal. Moreover, the Jacobian of the transformation $\left\{q_{\mu \alpha}\right\} \rightarrow\left\{\zeta_{\alpha}^{\text {rot }}, \zeta_{\kappa}\right\}$ is $J=\Pi_{\alpha} \sqrt{I_{\alpha \alpha}^{0}}$, where the coordinates $\zeta_{\kappa}$ are also orthonormal. Note that when the reference structure becomes linear, the Jacobian vanishes since one of the principal axes, e.g., $x$, lies along the molecular axis and, according to Eq. (29), it results that $\left\langle\zeta_{x}^{\mathrm{rot}} \mid \zeta_{x}^{\mathrm{rot}}\right\rangle=I_{x x}^{0}=0$

\section{Consistency of the two methods}

We now have two ways of determining the local space of infinitesimal rectilinear vibrational coordinates. In the first method we construct coordinates that are invariant under rotations $\left\{\zeta_{\kappa}^{\text {vibr }}, \zeta_{\kappa \lambda}^{\text {vibr }}\right\}$ through Eqs. (22), (26), and (27), and with them we build appropriate linear combinations that span the vibrational space $\mathcal{V}^{\text {vibr }}$. In the second method we find the complement of the rotational space $\mathcal{V}^{\text {rot }}$ in $\mathcal{V}$, which is constructed from Eckart's conditions. These two approaches will be equivalent if $\mathcal{V}^{\text {vibr }}$ and $\mathcal{V}^{\text {rot }}$ are orthogonal.

We now proceed to show that the inner product of any element in $\mathcal{V}^{\text {vibr }}$ with any element in $\mathcal{V}^{\text {rot }}$ vanishes. From Eqs. (26)-(28), it follows that

$$
\begin{gathered}
\left\langle\zeta_{\kappa}^{\mathrm{vibr}} \mid \zeta_{x}^{\mathrm{rot}}\right\rangle=\sqrt{M}\left(-q_{\kappa y}^{0} q_{\kappa z}^{0}+q_{\kappa y}^{0} q_{\kappa z}^{0}\right)=0 \\
\left\langle\zeta_{\kappa}^{\mathrm{vibr}} \mid \zeta_{y}^{\mathrm{rot}}\right\rangle=\sqrt{M}\left(-q_{\kappa z}^{0} q_{\kappa x}^{0}+q_{\kappa z}^{0} q_{\kappa x}^{0}\right)=0 \\
\left\langle\zeta_{\kappa}^{\mathrm{vibr}} \mid \zeta_{z}^{\mathrm{rot}}\right\rangle=\sqrt{M}\left(-q_{\kappa x}^{0} q_{\kappa y}^{0}+q_{\kappa x}^{0} q_{\kappa y}^{0}\right)=0 \\
\left\langle\zeta_{\kappa \lambda}^{\mathrm{vibr}} \mid \zeta_{x}^{\mathrm{rot}}\right\rangle=\sqrt{M}\left(-q_{\lambda y}^{0} q_{\kappa z}^{0}+q_{\lambda z}^{0} q_{\kappa y}^{0}-q_{\kappa y}^{0} q_{\lambda z}^{0}+q_{\kappa z}^{0} q_{\lambda y}^{0}\right)=0 \\
\left\langle\zeta_{\kappa \lambda}^{\mathrm{vibr}} \mid \zeta_{y}^{\mathrm{rot}}\right\rangle=\sqrt{M}\left(-q_{\lambda x}^{0} q_{\kappa z}^{0}+q_{\lambda z}^{0} q_{\kappa x}^{0}-q_{\kappa x}^{0} q_{\lambda z}^{0}+q_{\kappa z}^{0} q_{\lambda x}^{0}\right)=0 \\
\left\langle\zeta_{\kappa \lambda}^{\mathrm{vibr}} \mid \zeta_{z}^{\mathrm{rot}}\right\rangle=\sqrt{M}\left(-q_{\lambda x}^{0} q_{\kappa y}^{0}+q_{\lambda y}^{0} q_{\kappa x}^{0}-q_{\kappa x}^{0} q_{\lambda y}^{0}+q_{\kappa y}^{0} q_{\lambda x}^{0}\right)=0
\end{gathered}
$$

for $\kappa, \lambda=1 \ldots, N-1$. Therefore, the subspace $\mathcal{V}^{\text {vibr }}$ spanned by coordinates $\zeta_{\kappa}^{v i b r}, \zeta_{\kappa \lambda}^{\text {vibr }}$ coincides with the space of infinitesimal vibrations defined through Eckart's conditions.

As is often quoted in the literature, Eckart's conditions determine the rotating frame and subsequently the vibrations by requiring that for small departures from the reference structure, the angular momentum in the rotating frame vanishes [13]. We see that, alternatively, vibrations can be obtained by singling out and separating the rotations as the departures from the reference structure that preserve bond lengths and angles. This statement allows us to determine the 
vibrational coordinates at any reference configuration, $\mathbf{q}_{\kappa}^{0}$, not necessarily at a stationary point of the PES. On the other hand, for the rotational coordinates, rather than using the infinitesimal rectilinear rotations that we derived in Eqs. (23) and (28), we employ the three Euler angles.

Finally, in order to calculate the rovibrational levels of any molecule, we must construct an orthonormal basis $\zeta_{k}$, $1 \leq k \leq 3 N-6$, in the vibrational space $\mathcal{V}^{\text {vibr }}$, using the GramSchmidt procedure or some other orthogonalization algorithm. This basis can be expanded in the original basis, $q_{\mu \alpha}$, according to

$$
\left|\zeta_{k}\right\rangle=\sum_{\mu, \alpha} A_{k, \mu \alpha}\left(\left\{\mathbf{q}_{\kappa}^{0}\right\}\right) q_{\mu \alpha},
$$

where the matrix elements $A_{k, \mu \alpha}\left(\left\{\mathbf{q}_{\kappa}^{0}\right\}\right)$ are functions of the reference geometry. In what follows we shall drop this explicit dependence to avoid cluttering the notation.

\section{E. Watson Hamiltonian}

The molecular Hamiltonian in terms of rectilinear (locally defined) vibrational coordinates and Euler angles was derived by Wilson and Howard [14] in 1936. In their pioneering work they computed the classical kinetic energy and obtained the quantum Hamiltonian through an extension of the Podolsky transformation $[13,15]$. Still, the resulting Hamiltonian was difficult to implement unless one assumed that the inertia tensor is constant (which is often a good approximation for low-lying vibrational states). In 1968, Watson succeeded in reducing the quantum Hamiltonian to a form that was significantly simpler [1]. Although in principle unnecessary, for the sake of simplicity we shall restrict the following discussion to states with zero total angular momentum $(J=0)$, where the nuclear wave function does not depend on the Euler angles. In that case the Watson Hamiltonian reads as

$$
\hat{H}_{W}^{J=0}=\frac{1}{2} \sum_{\alpha, \beta} \mu_{\alpha \beta} \hat{\pi}_{\alpha} \hat{\pi}_{\beta}+\frac{1}{2 M} \sum_{k=1}^{3 N-6} \hat{P}_{\zeta_{k}}^{2}+U+\mathcal{E}_{\zeta},
$$

where $\alpha, \beta(=x, y, z)$ in the rotating frame of reference. Matrix elements $\mu_{\alpha \beta}$ are the components of the effective reciprocal inertia tensor,

$$
\mu_{\alpha \beta}=\left(I^{\prime}\right)_{\alpha \beta}^{-1},
$$

where

$$
I_{\alpha \beta}^{\prime}=I_{\alpha \beta}-M \sum_{k, l, m=1}^{3 N-6} \xi_{m l}^{\beta} \xi_{k l}^{\alpha} \zeta_{m} \zeta_{k}
$$

and

$$
\xi_{k l}^{\gamma}=\sum_{\mu=1}^{N-1} \sum_{\alpha, \beta} \epsilon_{\alpha \beta \gamma} A_{k, \mu \alpha} A_{l, \mu \beta} .
$$

The function $\mathcal{E}_{\zeta}$ is the PES expressed in terms of the vibrational coordinates, and $U$ is the so-called Watson term, which is proportional to the trace of the effective reciprocal inertia tensor [Eq. (39)],

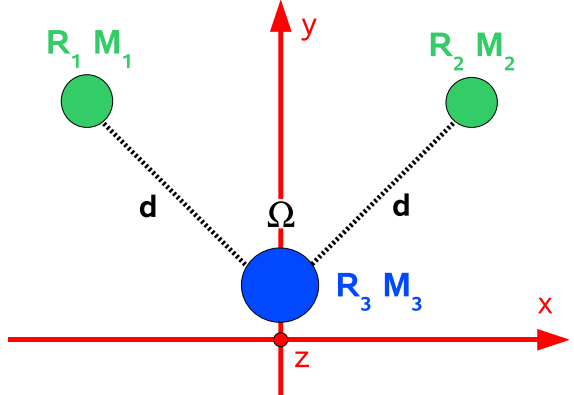

FIG. 1. (Color online) A triatomic $C_{2 v}$ molecule.

$$
U=-\frac{1}{8} \hbar^{2} \sum_{\alpha} \mu_{\alpha \alpha}
$$

Finally, $\hat{\pi}$ is the vibrational angular momentum, whose components are given by

$$
\hat{\pi}_{\alpha}=\sum_{l, k=1}^{3 N-6} \xi_{l k}^{\alpha} \zeta_{l} \hat{P}_{\zeta_{k}}
$$

where $\hat{P}_{\zeta_{k}}=-i \hbar \partial / \partial \zeta_{k}$. The volume element for integration is

$$
d V=(\sin \chi d \chi d \phi d \theta)\left(d \zeta_{1} d \zeta_{2} \cdots d \zeta_{3 N-6}\right),
$$

and $\sin \chi$ is the weight factor of the volume element [16]. It is important to note that the Watson Hamiltonian (38) is exact; no approximations have been introduced in its derivation and no limit is placed on the amplitude of the vibrations. With this, the solution of the Watson Hamiltonian should be independent of the choice of reference structure. We shall confirm that this is the case by calculating the vibrational spectrum of the water molecule with zero total angular momentum $(J=0)$. Nevertheless, the applicability of the Watson Hamiltonian to study triatomic molecules has been extensively questioned in the past [17]. The main reason is that the Watson Hamiltonian becomes singular at the linear configuration $[18,19]$. If one still wants to use the Watson Hamiltonian for triatomic molecules, then the linear configuration must not be sampled.

\section{EXAMPLE: GENERAL $\boldsymbol{C}_{2 v}$ TRIATOMIC MOLECULE}

In this section we shall consider the case of a general nonlinear triatomic molecule with $C_{2 v}$ symmetry, as shown in Fig. 1. We shall define the vibrational space for a reference structure respecting this symmetry but, apart from this, the reference geometry is arbitrary, and in general, it does not correspond to a stationary point in the PES.

We denote the nuclear masses by $M_{1}=M_{2}$ and $M_{3}$. The matrix $W_{\nu \nu^{\prime}}$ in Eq. (B5) of Appendix B is

$$
W=\left(\begin{array}{cc}
\frac{1}{M_{1}}+\frac{1}{M_{3}} & \frac{1}{M_{3}} \\
\frac{1}{M_{3}} & \frac{1}{M_{1}}+\frac{1}{M_{3}}
\end{array}\right) .
$$

The eigenvalues $w_{k}$ of $W$ are $w_{1}=\left(2 M_{1}+M_{3}\right) /\left(M_{1} M_{3}\right)$ and $w_{2}=1 / M_{1}$. For the corresponding eigenvectors $\left\langle\lambda_{k}\right|$ 
$=\left(\lambda_{k 1}, \lambda_{k 2}\right)$, we obtain $\lambda_{11}=\lambda_{12}$ and $\lambda_{21}+\lambda_{22}=0$. From Eq. (B1) we have $\lambda_{13}=-2 \lambda_{11}$ and $\lambda_{23}=0$. Therefore, the relative coordinates are

$$
\begin{gathered}
\mathbf{Q}_{1}=\lambda_{11}\left(\mathbf{R}_{1}+\mathbf{R}_{2}-2 \mathbf{R}_{3}\right), \\
\mathbf{Q}_{2}=\lambda_{12}\left(\mathbf{R}_{1}-\mathbf{R}_{2}\right) .
\end{gathered}
$$

From Eq. (B8) in Appendix B, the inverse of the masses are $1 / m_{1}=2 w_{1}\left(\lambda_{11}\right)^{2}$ and $1 / m_{2}=2 w_{2}\left(\lambda_{21}\right)^{2}$. The constants $\lambda_{11}$ and $\lambda_{21}$ must be chosen so that the Jacobian of the transformation to the c.m. and relative coordinates (1)-(3) is unity. We choose $\lambda_{11}=-1 / 2, \lambda_{21}=1$ and obtain the well-known Jacobi scattering coordinates for the relative coordinates,

$$
\begin{gathered}
\mathbf{Q}_{1}=\mathbf{R}_{3}-\frac{\mathbf{R}_{1}+\mathbf{R}_{2}}{2}, \\
\mathbf{Q}_{2}=\mathbf{R}_{1}-\mathbf{R}_{2} .
\end{gathered}
$$

The coefficients $\lambda_{\mu n}$ given by Eq. (3) are

$$
\begin{gathered}
\lambda_{11}=-\frac{1}{2}, \quad \lambda_{12}=-\frac{1}{2}, \quad \lambda_{13}=1, \\
\lambda_{21}=1, \quad \lambda_{22}=-1, \quad \lambda_{23}=0
\end{gathered}
$$

so that expression (5) is fulfilled. The transformation of course leads to diagonal $\beta_{\kappa \kappa}$ as defined in Eq. (11);

$$
\begin{gathered}
\beta_{11}=\frac{1}{m_{1}}=\frac{2 M_{1}+M_{3}}{2 M_{1} M_{3}}, \\
\beta_{22}=\frac{1}{m_{2}}=\frac{2}{M_{1}},
\end{gathered}
$$

while the coefficients $\gamma_{\mu n}$ [Eq. (6)] are

$$
\begin{gathered}
\gamma_{11}=\frac{M_{3}}{2 M_{1}+M_{3}}, \quad \gamma_{21}=-\frac{M_{1}}{2 M_{1}+M_{3}}, \\
\gamma_{12}=\frac{M_{3}}{2 M_{1}+M_{3}}, \quad \gamma_{22}=\frac{M_{1}}{2 M_{1}+M_{3}}, \\
\gamma_{13}=-\frac{2 M_{1}}{2 M_{1}+M_{3}}, \quad \gamma_{23}=0
\end{gathered}
$$

and the mass-scaling transformation [Eq. (18)] gives for the common mass

$$
M=M_{1} \sqrt{\frac{M_{3}}{2 M_{1}+M_{3}}} .
$$

According to Eq. (22), the vibrational coordinates must be linear combinations of $\mathbf{q}_{\mu}^{0} \cdot \mathbf{q}_{\kappa}(\mu, \kappa=1,2)$. We choose for the first vibrational coordinate,

$$
\zeta_{1}=\frac{1}{L}\left(\mathbf{q}_{1}^{0} \cdot \mathbf{q}_{1}+\mathbf{q}_{2}^{0} \cdot \mathbf{q}_{2}\right),
$$

with

$$
L=\sqrt{\left|\mathbf{q}_{1}^{0}\right|^{2}+\left|\mathbf{q}_{2}^{0}\right|^{2}} .
$$

For $\zeta_{2}$ we choose a linear combination of $\mathbf{q}_{1}^{0} \cdot \mathbf{q}_{1}$ and $\mathbf{q}_{2}^{0} \cdot \mathbf{q}_{2}$ that is orthogonal to $\zeta_{1}$

$$
\zeta_{2}=\frac{1}{L}\left(\frac{\left|\mathbf{q}_{2}^{0}\right|}{\left|\mathbf{q}_{1}^{0}\right|} \mathbf{q}_{1}^{0} \cdot \mathbf{q}_{1}-\frac{\left|\mathbf{q}_{1}^{0}\right|}{\left|\mathbf{q}_{2}^{0}\right|} \mathbf{q}_{2}^{0} \cdot \mathbf{q}_{2}\right) .
$$

Finally, $\zeta_{3}$ is defined by the component of $\left(\mathbf{q}_{2}^{0} \cdot \mathbf{q}_{1}\right.$ $\left.+\mathbf{q}_{1}^{0} \cdot \mathbf{q}_{2}\right) / L$ orthogonal to $\zeta_{1}$ and $\zeta_{2}$. Since, for a $C_{2 v}$ reference, $\mathbf{q}_{1}^{0} \cdot \mathbf{q}_{2}^{0}=0$, we obtain

$$
\zeta_{3}=\frac{1}{L}\left(\mathbf{q}_{2}^{0} \cdot \mathbf{q}_{1}+\mathbf{q}_{1}^{0} \cdot \mathbf{q}_{2}\right) .
$$

Vibrational coordinate $\zeta_{3}$ is linearly independent from the other two except when the reference configuration becomes linear.

With these definitions we can construct matrix $A$ with elements $A_{k, \mu \alpha}, k=1,2,3, \mu=1,2$, and $\alpha=x, y, z$ [Eq. (37)] as follows:

$$
A=\frac{1}{L}\left(\begin{array}{cc}
\mathbf{q}_{1}^{0} & \mathbf{q}_{2}^{0} \\
\frac{\left|\mathbf{q}_{2}^{0}\right|}{\left|\mathbf{q}_{1}^{0}\right|} \mathbf{q}_{1}^{0} & -\frac{\left|\mathbf{q}_{1}^{0}\right|}{\left|\mathbf{q}_{2}^{0}\right|} \mathbf{q}_{2}^{0} \\
\mathbf{q}_{2}^{0} & \mathbf{q}_{1}^{0}
\end{array}\right) .
$$

Note the shorthand vector notation for the $3 \times 6$ matrix $A$ : the symbol $\mathbf{q}_{1}^{0}$ stands for $q_{1 x}^{0}, q_{1 y}^{0}, q_{1 z}^{0}$, and similarly for $\mathbf{q}_{2}^{0}$. Coordinates $\zeta_{1}, \zeta_{2}$, and $\zeta_{3}$ are close to the usual normal modes, corresponding to symmetric stretching, bending, and asymmetric stretching motions, respectively.

Since any nonlinear triatomic molecule is planar, the orientation of the rotating frame of reference is chosen such that the problem is described in the $x y$ plane. With this, the $z$ values of the nuclear coordinates are zero. From Eq. (41), it follows that

$$
\begin{gathered}
\xi_{l k}^{x}=\xi_{l k}^{y}=0, \quad l, k=1,2,3, \\
\xi_{12}^{z}=\xi_{21}^{z}=0, \\
\xi_{13}^{z}=\xi_{31}^{z}=0, \\
\xi_{23}^{z}=-\xi_{32}^{z}=1,
\end{gathered}
$$

and the components of the vibrational angular momentum [Eq. (43)] are

$$
\begin{gathered}
\hat{\pi}_{x}=0, \\
\hat{\pi}_{y}=0, \\
\hat{\pi}_{z}=\zeta_{2} \hat{P}_{\zeta_{3}}-\zeta_{3} \hat{P}_{\zeta_{2}} .
\end{gathered}
$$

The components of the effective inertia tensor $I_{\alpha \beta}^{\prime}[\mathrm{Eq}$. (40)] are

$$
I_{\alpha \beta}^{\prime}=I_{\alpha \beta}\left(1-\delta_{\alpha z} \delta_{\beta z}\right)+\delta_{\alpha z} \delta_{\beta z}\left[I_{z z}-M\left(\zeta_{2}^{2}+\zeta_{3}^{2}\right)\right],
$$




$$
\alpha, \beta=x, y, z,
$$

where $M$ is given by Eq. (53) and $I_{\alpha \beta}$ is a function of the vibrational coordinates $\zeta_{1}, \zeta_{2}$, and $\zeta_{3}$. Finally, the Watson Hamiltonian for a triatomic $C_{2 v}$ molecule with $J=0$ reads as

$$
\hat{H}_{W}^{J=0}=\frac{1}{2} \mu_{z z} \hat{\pi}_{z} \hat{\pi}_{z}+\frac{1}{2 M} \sum_{k=1}^{3} \hat{P}_{\zeta_{k}}^{2}+U+\mathcal{E}_{\zeta} .
$$

\section{Application: The water molecule}

The study of the rovibrational spectra of water has always been an interesting challenge [20-24]. This nonlinear semirigid molecule has a high barrier to linearity $\left(\approx 11000 \mathrm{~cm}^{-1}\right)[25]$. The first calculation of highly excited states for $J=0$ within the framework of the Watson Hamiltonian was performed in 1998 by Bowman and Carter [26]. These results were obtained with the MULTIMODE code [26-29]. They also performed calculations for $J=1, J=3$, and $J=5$. Mátyus et al. [19] proposed an effective general algorithm for variational vibrational calculations using global, orthogonal, and rectilinear internal coordinates. The matrix representation of the Watson Hamiltonian was constructed using a Hermite-discrete variable representation (DVR) basis. As a test of their algorithm, they reported the first 21 vibrational states of the water molecule for $J=0$.

Here, we implemented the exact diagonalization of the Watson Hamiltonian using the Lagrange-mesh method $[30,31]$ for the construction of the matrix elements. The Lagrange grids used were based on the Cartesian orthogonal polynomials [32].

In order to test the validity of the present methodology for the definition of local rectilinear vibrational coordinates, we solve the full Watson Hamiltonian exactly for different reference structures. Since the Watson Hamiltonian is exact, the spectrum should not depend on the choice of rectilinear vibrational coordinates (or, equivalently, on the choice of the reference structure). However, the results will be incorrect if the set of coordinates does not correspond to pure vibrations, i.e., if it is inconsistent with the reference configuration. Evidently, if rotations and vibrations are mixed, the Watson Hamiltonian is not a valid description of the problem.

For the water molecule, we used the PES constructed by Partridge and Schwenke [33]. The minimum energy structure corresponds to a configuration with $d_{\mathrm{OH}}=1.81$ bohr and internal angle $\Omega=104.44^{\circ}$. We will take the solution of the Watson Hamiltonian for this reference configuration as our target. With the exception of a few states, our target results were in excellent agreement both, with the results reported by Bowman and Carter [26] and by Partridge and Schwenke [33], who employed Radau curvilinear internal coordinates. For nonlinear triatomic molecules, because of the singularity of the Jacobian of the transformation and the ensuing singularity of the inverse moment of inertia in the Watson Hamiltonian, we consider the results in terms of Radau coordinates to be superior and effectively "exact" [26].

For the few states (i.e., states 15, 20, 24, 32, and 34) where all three sets of frequencies differed-our mean deviation from "exact" results was $54 \mathrm{~cm}^{-1}$, while in Ref. [26] it was $238 \mathrm{~cm}^{-1}$, convergence was very slow, thus casting doubts on the level of convergence attained and the quality of those particular results.

We then considered two arbitrary nonstationary reference configurations: (a) $d_{\mathrm{OH}}=2$ bohr, $\Omega=120^{\circ}$ and (b) $d_{\mathrm{OH}}$ $=1.8 \mathrm{bohr}, \Omega=90^{\circ}$. For these reference structures we solved the Watson Hamiltonian using (deliberately wrong) the vibrational coordinates corresponding to the minimum of the PES. Figure 2 shows the differences of the resulting spectra with respect to the target spectrum, which was computed with the reference geometry at the minimum of the PES. Clearly, the magnitude of the energy differences is unacceptable, thus highlighting the importance of the correct definition of vibrational coordinates in the description of the problem.

For the same nonstationary references we solved the Watson Hamiltonian again, this time using the appropriate vibrational coordinates given by Eq. (58), which are consistent with the chosen reference configuration. The energy differences with respect to the target spectrum are shown in Fig. 3. First, it is important to notice the difference in scale between the two figures. The energy differences drop in more than 3 orders of magnitude. In fact, for many states both black squares and red circles essentially coincide with the green triangles. Therefore, we conclude that a major improvement is obtained with respect to the previous results (Fig. 2).

In Fig. 3 we do not report the results for states 15, 20, 24, 32 , and 34 , as for these states we have not succeeded in obtaining satisfactory results using nonstationary reference structures. The behavior with increasing number of grid points proved oscillatory and convergence extremely slow. Interestingly, these are the same states mentioned above for which our results and those in Ref. [26] differed significantly from the "exact" ones [33]. Mátyus et al. [19], who solved the Watson Hamiltonian for water on a different PES, also reported slow convergence for states 15 and 20. Both these studies used the equilibrium geometry as their reference structure.

Based on the results of Figs. 2 and 3, we conclude that the definition of vibrational coordinates proposed here is sound and valid at any point of the PES. In addition, previous $[19,26]$ and present results obtained with the Watson Hamiltonian compare very well with exact results [33]. There are, however, few states for which the Watson Hamiltonian does not provide a good description compared with the exact ones. These are characterized by an unsurmountable difficulty in reaching convergence as the number of grid points is increased. This is an inherent limitation of the Watson Hamiltonian for describing highly excited states of the bending coordinate in the water molecule [34]. In fact, this degree of freedom brings the system toward the linear configuration, and therefore, the near-singular region of the Watson Hamiltonian operator is sampled.

\section{CONCLUSIONS}

The aim of this work was to provide a general method for defining local rectilinear vibrational coordinates at arbitrary reference structures since for highly anharmonic systems the 


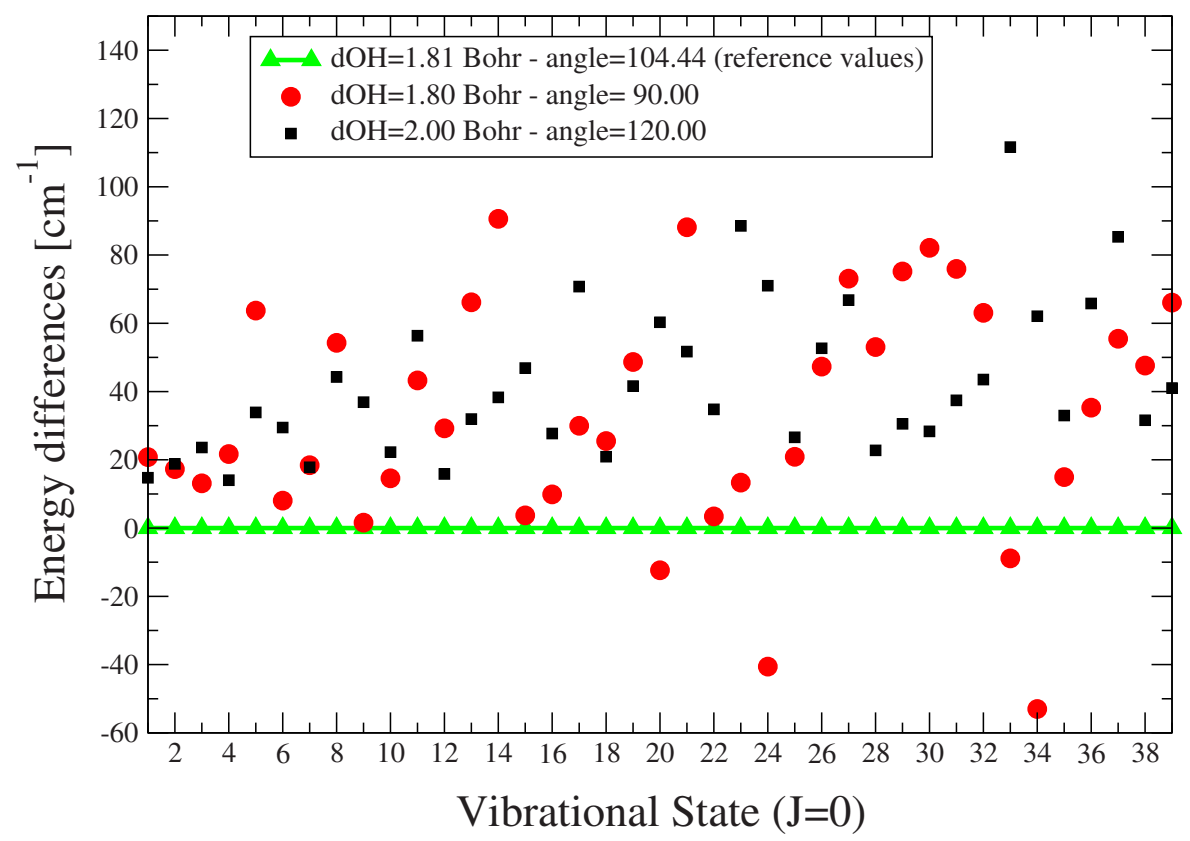

FIG. 2. (Color online) Effect of the choice of vibrational space on the vibrational spectrum. The spectrum obtained with the reference geometry at the minimum of the PES is taken as the target (green triangles). Black squares and red circles denote energy differences with respect to the target values for two different nonstationary configurations (see legend). For each configuration, we solve the Watson Hamiltonian using the vibrational space corresponding to the minimum of the PES. Clearly, the use of an incorrect vibrational space leads to a poor description of the problem.

most efficient structure for the solution of the Watson Hamiltonian is not necessarily the equilibrium one. To this end, we developed a methodology and tested it by applying the Watson Hamiltonian to compute the vibrational spectrum of wa- ter molecule with zero angular momentum $(J=0)$.

We verified analytically and numerically that these coordinates are consistent with the Eckart's conditions that define the rotating frame of reference. We also showed that the

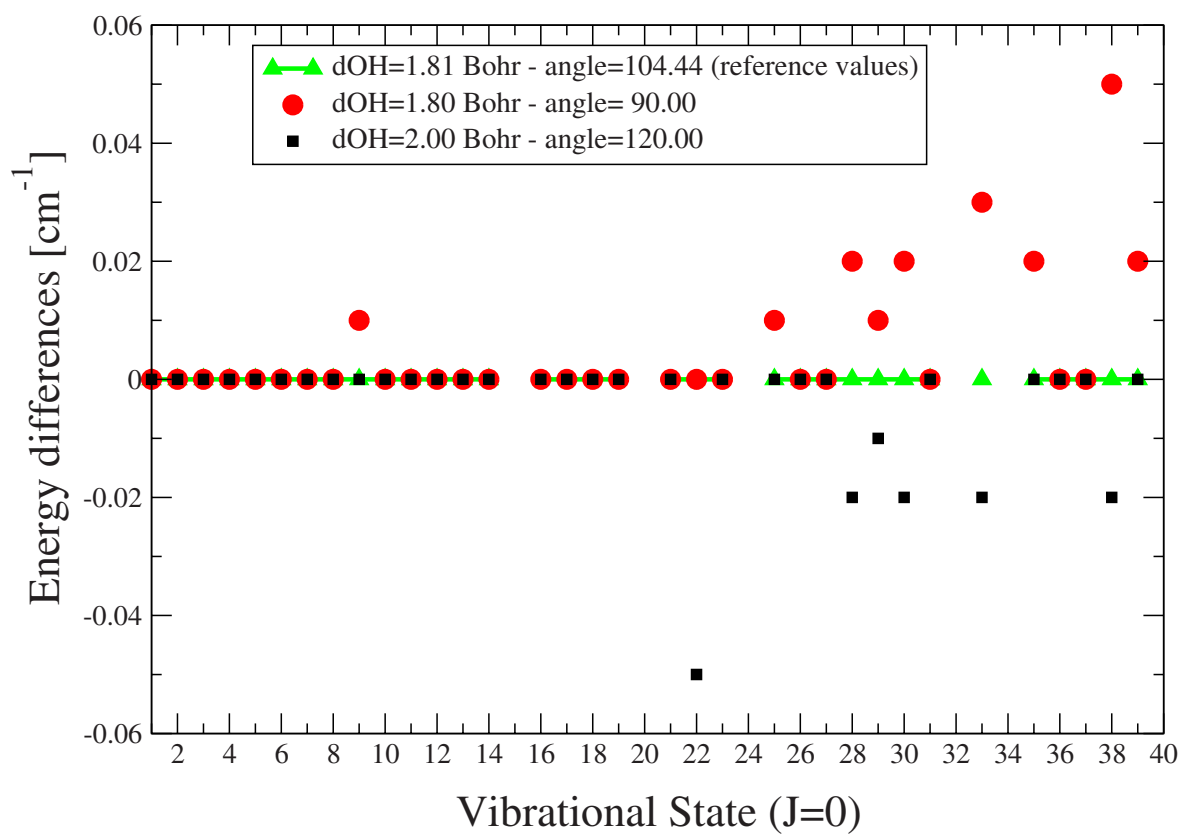

FIG. 3. (Color online) Effect of the reference configuration on the vibrational spectrum. The spectrum obtained with the reference geometry at the minimum of the PES is taken as the target (green triangles). Black squares and red circles denote energy differences with respect to the reference values, for two different nonstationary configurations. For each reference geometry, the vibrational space is obtained from Eq. (58). We have avoided the calculation of states 15, 20, 24, 32, and 34 as they proved difficult to converge with the number of grid points. Comparing with Fig. 2 we can state that the results are essentially independent of the reference configuration, within numerical accuracy. 
Watson Hamiltonian can be applied to the description of vibrational spectra of triatomic molecules using any arbitrary reference structure. The dependence on the reference structure is exactly eliminated by the inclusion of the vibrational angular momentum and the Watson terms in Eq. (38). In fact, when these terms are neglected, as in some approximate schemes, the choice of the reference structure may become an important issue, especially for small molecules. It is important to bear in mind that the formalism of the Watson Hamiltonian has limitations for the calculation of certain high-quanta bending states of triatomic molecules. These states, however, can be readily identified through slow or lack of convergence. Although it is widely agreed that the use of the Watson Hamiltonian is not convenient for describing triatomic molecules [34], this is not the case of tetratomic and larger systems, where the use of local rectilinear vibrational coordinates is actively being pursued [35].

\section{ACKNOWLEDGMENTS}

We are grateful to B. T. Sutcliffe and J. Bowman for enlightening discussions. I.S. acknowledges helpful correspondence by J. K. G. Watson, A. G. Császár, J. Tennyson, and S. Carter.

\section{APPENDIX A: SOME PROPERTIES OF $\lambda_{\kappa n}$ AND $\gamma_{\kappa n}$}

Multiplying both sides of Eq. (6) by $\lambda_{\kappa n}$ and summing over particles, we obtain

$$
\sum_{n=1}^{N} \lambda_{\kappa n} \mathbf{R}_{n}=\mathbf{R}_{\mathrm{c} . \mathrm{m} .} \sum_{n=1}^{N} \lambda_{\kappa n}+\sum_{\mu=1}^{N-1} \mathbf{Q}_{\mu} \sum_{n=1}^{N} \lambda_{\kappa n} \gamma_{\mu n},
$$

and therefore,

$$
\begin{gathered}
\mathbf{Q}_{\kappa}=\sum_{\mu=1}^{N-1} \mathbf{Q}_{\mu} \sum_{n=1}^{N} \lambda_{\kappa n} \gamma_{\mu n} \\
\Rightarrow \sum_{n=1}^{N} \lambda_{\kappa n} \gamma_{\mu n}=\delta_{\kappa \mu} .
\end{gathered}
$$

On the other hand, multiplying both sides of Eq. (3) by $\gamma_{\mu n}$ and summing over relative coordinates, it follows that

$$
\begin{gathered}
\sum_{\mu=1}^{N-1} \gamma_{\mu n} \mathbf{Q}_{\mu}=\sum_{l=1}^{N} \mathbf{R}_{l} \sum_{\mu=1}^{N-1} \gamma_{\mu n} \lambda_{\mu l} \\
\Rightarrow \mathbf{R}_{n}-\mathbf{R}_{\mathrm{c} . \mathrm{m} .}=\sum_{l=1}^{N} \mathbf{R}_{l} \sum_{\mu=1}^{N-1} \gamma_{\mu n} \lambda_{\mu l} \\
\Rightarrow \sum_{\mu=1}^{N-1} \gamma_{\mu n} \lambda_{\mu l}=\delta_{l n}-\frac{M_{l}}{M_{\mathrm{c} . \mathrm{m} .}} .
\end{gathered}
$$

We consider now the condition for diagonal $\beta_{\mu \kappa}$,

$$
\sum_{n=1}^{N} \frac{\lambda_{\mu n} \lambda_{\kappa n}}{M_{n}}=\frac{\delta_{\mu \kappa}}{m_{\kappa}} .
$$

From Eq. (A6) we obtain

$$
\begin{gathered}
\sum_{l=1}^{N} \sum_{\mu=1}^{N-1} \gamma_{\mu n} \lambda_{\mu l} \frac{\lambda_{\kappa l}}{M_{l}}=\sum_{l=1}^{N}\left(\delta_{l n}-\frac{M_{l}}{M_{\text {c.m. }}}\right) \frac{\lambda_{\kappa l}}{M_{l}} \\
\Rightarrow \frac{\gamma_{\kappa n}}{m_{\kappa}}=\frac{\lambda_{\kappa n}}{M_{n}} .
\end{gathered}
$$

This is the main relation connecting $\gamma_{\kappa n}$ and $\lambda_{\kappa n}$. Equation (A7) becomes

$$
\begin{aligned}
& \sum_{n=1}^{N} M_{n} \frac{\lambda_{\mu n}}{M_{n}} \frac{\lambda_{\kappa n}}{M_{n}}=\frac{\delta_{\mu \kappa}}{m_{\kappa}} \\
\Rightarrow & \sum_{n=1}^{N} M_{n} \frac{\gamma_{\mu n}}{m_{\mu}} \frac{\gamma_{\kappa n}}{m_{\kappa}}=\frac{\delta_{\mu \kappa}}{m_{\kappa}} \\
\Rightarrow & \sum_{n=1}^{N} M_{n} \gamma_{\mu n} \gamma_{\kappa n}=m_{\kappa} \delta_{\mu \kappa} .
\end{aligned}
$$

\section{APPENDIX B: A GENERAL METHOD TO OBTAIN A SET OF RELATIVE COORDINATES WITH DIAGONAL $\boldsymbol{\beta}_{\mu \kappa}$}

From Eq. (5), we obtain

$$
\lambda_{\mu N}=-\sum_{n=1}^{N-1} \lambda_{\mu n}, \quad \mu=1 \ldots N-1,
$$

and from Eq. (11) we have

$$
\sum_{n=1}^{N} \frac{1}{M_{n}} \lambda_{\mu n} \lambda_{\kappa n}=0, \quad \mu \neq \kappa .
$$

Substituting Eq. (B1) in the above expression, we obtain

$$
\sum_{\nu=1}^{N-1} \frac{1}{M_{\nu}} \lambda_{\mu \nu} \lambda_{\kappa \nu}+\frac{1}{M_{N}} \sum_{\nu \nu^{\prime}=1}^{N-1} \lambda_{\mu \nu} \lambda_{\kappa \nu^{\prime}}=0, \quad \mu \neq \kappa,
$$

or

$$
\sum_{\nu \nu^{\prime}=1}^{N-1} \lambda_{\mu \nu} W_{\nu \nu^{\prime}} \lambda_{\kappa \nu^{\prime}}=0, \quad \mu \neq \kappa
$$

with

$$
W_{\nu \nu^{\prime}}=\frac{\delta_{\nu \nu^{\prime}}}{M_{\nu}}+\frac{1}{M_{N}}>0 .
$$

We can see that Eq. (B4) is a matrix equation,

$$
\left\langle\lambda_{\mu}|W| \lambda_{\kappa}\right\rangle=0, \quad \mu \neq \kappa,
$$

with $\left\langle\lambda_{\mu}\right|=\left(\lambda_{\mu 1}, \lambda_{\mu 2}, \ldots, \lambda_{\mu N-1}\right)$ and $W_{\nu \nu^{\prime}}$ the matrix elements of the $(N-1) \times(N-1)$ matrix $W$ defined in Eq. (B5). 
Therefore, a way to satisfy Eq. (B4) is to choose $\left|\lambda_{\kappa}\right\rangle$ to be the eigenvectors of $W$

$$
W\left|\lambda_{\kappa}\right\rangle=w_{\kappa}\left|\lambda_{\kappa}\right\rangle .
$$

Since matrix $W$ is positive definite, its eigenvalues $\left(w_{\kappa}\right)$ are positive. With this, Eq. (11) gives

$$
\beta_{\mu \kappa}=\delta_{\mu \kappa} w_{\kappa}\left\langle\lambda_{\kappa} \mid \lambda_{\kappa}\right\rangle
$$

where

$$
\left\langle\lambda_{\kappa} \mid \lambda_{\kappa}\right\rangle=\sum_{\nu=1}^{N-1}\left(\lambda_{\kappa \nu}\right)^{2} .
$$

According to Eq. (12), the mass of the relative coordinates $\mathbf{Q}_{\kappa}$ is $m_{\kappa}^{-1}=w_{\kappa}\left\langle\lambda_{\kappa} \mid \lambda_{\kappa}\right\rangle$. The normalization of vectors $\left|\lambda_{\kappa}\right\rangle$, given by Eq. (B9), must be determined by requiring that the Jacobian of the transformation [Eqs. (1)-(3)] be unity while, in general, $\left\langle\lambda_{\kappa} \mid \lambda_{\kappa}\right\rangle \neq 1$.
[1] J. K. G. Watson, Mol. Phys. 15, 479 (1968).

[2] J. K. G. Watson, Mol. Phys. 19, 465 (1970).

[3] C. Eckart, Phys. Rev. 47, 552 (1935).

[4] J. D. Louck and H. W. Galbraith, Rev. Mod. Phys. 48, 69 (1976).

[5] J. K. G. Watson, private communication.

[6] For systems with weak or moderate anharmonicity the difference between the classical and quantum-averaged structures is small.

[7] C. Léonard, N. C. Handy, S. Carter, and J. M. Bowman, Spectrochim. Acta, Part A 58, 825 (2002).

[8] R. B. Gerber and O. J. Joon, Computational Molecular Spectroscopy (Wiley, NY, 2000), Chap. 11.

[9] M. A. Ratner and R. B. Gerber, J. Phys. Chem. 90, 20 (1986).

[10] A. Roitberg, R. B. Gerber, R. Elber, and M. A. Ratner, Science 268, 1319 (1995).

[11] I. Scivetti, J. Kohanoff, and N. Gidopoulos (unpublished).

[12] M. Born and J. R. Oppenheimer, Ann. Phys. 389, 457 (1927).

[13] E. B. Wilson, Jr., J. C. Decius, and P. C. Cross, Molecular Vibrations (McGraw-Hill, NY, 1955).

[14] E. B. Wilson, Jr. and J. B. Howard, J. Chem. Phys. 4, 260 (1936).

[15] B. Podolsky, Phys. Rev. 32, 812 (1928).

[16] H. Meyer, Annu. Rev. Phys. Chem. 53, 141 (2002).

[17] M. J. Bramley and T. Carrington, Jr., J. Chem. Phys. 99, 8519 (1993).

[18] R. Bartholomae, D. Martin, and B. T. Sutcliffe, J. Mol. Spectrosc. 87, 367 (1981).

[19] E. Mátyus, G. Czakó, B. T. Sutcliffe, and A. G. Császár, J.
Chem. Phys. 127, 084102 (2007).

[20] M. G. Bucknell and N. C. Handy, Mol. Phys. 28, 777 (1974).

[21] B. T. Sutcliffe and J. Tennyson, J. Chem. Soc., Faraday Trans. 2 83, 1663 (1987).

[22] R. J. Whitehead and N. C. Handy, J. Mol. Spectrosc. 55, 356 (1975); J. Mol. Spectrosc. 59, 459 (1976).

[23] G. J. Sexton and N. C. Handy, Mol. Phys. 51, 1321 (1984).

[24] C.-L. Chen, B. Maessen, and M. Wolfsberg, J. Chem. Phys. 83, 1795 (1985).

[25] P. R. Bunker and J. M. R. Stone, J. Mol. Spectrosc. 41, 310 (1972).

[26] S. Carter and J. M. Bowman, J. Chem. Phys. 108, 4397 (1998).

[27] S. Carter, S. J. Culik, and J. M. Bowman, J. Chem. Phys. 107, 10458 (1997)

[28] S. Carter and J. M. Bowman, J. Phys. Chem. A 104, 2355 (2000).

[29] J. Dai, Z. Bačić, X. Huang, S. Carter, and J. M. Bowman, J. Chem. Phys. 119, 6571 (2003).

[30] D. Baye and P.-H. Heenen, J. Phys. A 19, 2041 (1986); D. Baye, Phys. Status Solidi B 243, 1095 (2006).

[31] K. Varga, Z. Zhang, and S. T. Pantelides, Phys. Rev. Lett. 93, 176403 (2004).

[32] T. Seideman and W. H. Miller, J. Chem. Phys. 97, 2499 (1992).

[33] H. Partridge and D. W. Schwenke, J. Chem. Phys. 106, 4618 (1997).

[34] A. G. Császár, private communication.

[35] J. Tennyson, Computational Molecular Spectroscopy (Wiley, NY, 2000), Chap. 9. 